\title{
L'immigration japonaise en Nouvelle-Calédonie : une illustration de l'affirmation du Japon dans le Pacifique
}

\section{Yann Bencivengo}

\section{(2) OpenEdition \\ Journals}

Édition électronique

URL : http://journals.openedition.org/jso/6725

DOI : $10.4000 /$ jso. 6725

ISSN : $1760-7256$

Éditeur

Société des océanistes

\section{Édition imprimée}

Date de publication : 31 décembre 2012

Pagination : 215-228

ISBN : 978-2-85430-033-8

ISSN : 0300-953x

Référence électronique

Yann Bencivengo, «L'immigration japonaise en Nouvelle-Calédonie : une illustration de l'affirmation du Japon dans le Pacifique », Journal de la Société des Océanistes [En ligne], 135 | 2012-2, mis en ligne le 18 février 2013, consulté le 30 avril 2019. URL : http://journals.openedition.org/jso/6725 ; DOI : $10.4000 /$ jso. 6725 


\title{
L'immigration japonaise en Nouvelle-Calédonie : une illustration de l'affirmation du Japon dans le Pacifique
}

par

\author{
Yann BENCIVENGO*
}

\section{RÉSUMÉ}

De 1892 à 1919, environ 5500 travailleurs japonais ont été recrutés par des compagnies minières en Nouvelle-Calédonie. Au premier rang d'entre elles se trouve la société Le Nickel qui est à l'origine du premier recrutement de Japonais dans la colonie française. Ce mouvement migratoire présente des caractéristiques originales par rapport à l'ensemble des migrations de travail qui traversent alors le Pacifique. Le Japon entend bien que ses ressortissants soient traités et considérés comme des travailleurs libres à pied d'égalité avec les Européens. Les engagés japonais, réputés efficaces, font preuve d'une grande pugnacité dans la défense de leurs droits et de modernité dans leur comportement vis-à-vis de leurs employeurs. Ils n'hésitent pas à se lancer dans des grèves ou à rompre leur contrat afin de trouver une meilleure situation. L'âpreté des négociations menées pour obtenir ces travailleurs comme le comportement de ces derniers reflètent l'accession du Japon au rang de grande puissance de l'espace Asie-Pacifique. Cette émigration marque aussi le début de l'entrée de la NouvelleCalédonie dans l'espace économique régional.

Mots-CLÉS : Nouvelle-Calédonie, Japon, migrations, relations internationales, nickel, main-d'œuvre, activité minière

Pendant la deuxième moitié du XIX ${ }^{\mathrm{e}}$ siècle et le début du suivant, le Pacifique est traversé par de nombreux flux migratoires de travailleurs : coolies chinois importés à Tahiti, Néo-Hébridais employés au Queensland ou en Nouvelle-

\section{ABSTRACT}

From 1892 to 1919, about 5,500 Japanese workers were hired by mining companies in New Caledonia. At the forefront of them is Societe Le Nickel which is at the origin of first recruitment of Japanese in the French colony. This migration has unique features compared to the overall labor migration while crossing the Pacific. Japan intends that its citizens are treated and considered as free workers on an equal footing with the Europeans. The Japanese committed, deemed effective, show great tenacity in defense of their rights and modernity in their behavior vis-à-vis their employers. They do not hesitate to engage in strikes or break their contract in order to find a better situation. The harshness of the negotiations for these workers as the behavior of these reflect the accession of Japan as a great power of Asia-Pacific region. This migration also marks the beginning of the entry of New Caledonia in the regional economic space.

KEYwORDs: New Caledonia, Japan, migration, international relations, nickel, labor, mining

Calédonie, Indiens venus par dizaines de milliers travailler sur les plantations de Fidji, etc. Ces migrations peuvent être replacées dans le cadre du blackbirding, c'est-à-dire d'un mode de recrutement dont certains aspects s'apparentent à

* Chercheur-associé au CH2ST (EA I27), Université de Paris 1 Panthéon-Sorbonne, yann.benci@wanadoo.fr 
l'esclavagisme, notamment en raison du caractère forcé qu'il a fréquemment présenté. Les migrations de travailleurs japonais vers Hawaii et la Nouvelle-Calédonie s'en distinguent. D'une part, le gouvernement japonais veille à ce que ses ressortissants soient décemment traités tout en s'attachant à exercer sur eux une surveillance soutenue. D'autre part, les engagés japonais ont une qualification et un comportement qui tranchent souvent avec ceux des autres migrants.

En ce qui concerne la Nouvelle-Calédonie, le livre de Kobayashi Tadao (1992) constitue l'ouvrage de référence de l'histoire de ces émigrants. Plusieurs travaux sont venus le compléter portant sur des sujets plus larges (Palombo, 2002) ou ciblant tel aspect ou tel épisode de la présence des Japonais dans la colonie. En 20062007, une exposition intitulée " Feu nos pères », consacrée aux immigrés japonais et à leurs descendants, a été présentée par l'artiste Mutsumi Tsuda au centre Tjibaou de Nouméa, puis en itinérance au Japon. Ici, il s'agit, à partir de l'étude de la main-d'œuvre japonaise employée par la société Le Nickel en Nouvelle-Calédonie de 1892jusqu'au lendemain dela Première Guerre mondiale, de replacer ce mouvement migratoire dans un contexte plus général, de cerner les motivations qui ont conduit à son établissement, de décrire ses principales caractéristiques et de comprendre en quoi il est révélateur de la nouvelle place prise par le Japon sur la scène internationale en ce début de $\mathrm{xx}^{\mathrm{e}}$ siècle.

\section{Une initiative privée}

La Nouvelle-Calédonie se révèle être une terre minière dans les vingt années qui suivent sa prise de possession par la France en 1853 : or, cuivre, cobalt, chrome et surtout nickel y sont extraits. C'est à partir de 1875 que les minerais de nickel calédoniens commencent à être exploités. En 1880 , les principaux gisements entrent dans le giron de la société Le Nickel, une nouvelle société minière et métallurgique, créée à Paris, qui passe trois ans plus tard sous le contrôle de la banque Rothschild. La société Le Nickel domine alors largement l'économie minière locale'.

Il apparaît rapidement que les gisements peuvent être travaillés à ciel ouvert. Dès lors, il est possible d'utiliser une main-d'œuvre peu qualifiée dont le travail s'apparente à un travail de terrassement. Mais, comme dans de nombreux autres pays du Pacifique, la Nouvelle-Calédonie est confrontée au manque de main-d'œuvre et, en 1883 , un sénateur de retour de mission en Nouvelle-Calédonie écrit :

« De la main-d'œuvre! Des travailleurs! Tel est le cri qui retentit d'un bout à l'autre de l'Océan Pacifique. » (Archives de l'Outre-Mer, série géographique, carton 180, rapport Courmeaux)

La société Le Nickel emploie alors des NéoHébridais pour compléter ses effectifs. Cette source de main-d'œuvre s'est assez rapidement tarie en raison des abus commis par les recruteurs. Suspendu une première fois en 1882-1883, ce mouvement d'immigration est de nouveau interdit de 1885 à 1890. En outre, la mauvaise réputation du travail des mines dissuade les Néo-Hébridais d'accepter d'être engagés dans la colonie française. En raison de la présence d'un bagne et de son entregent auprès du gouvernement français, la société Le Nickel peut alors bénéficier de la main-d'œuvre pénale, nombreuse et très peu chère, qu'elle se procure grâce à des contrats très avantageux. Cependant, pour répondre à l'augmentation de la demande en nickel qui se dessine à la fin des années 1880, il lui faut trouver d'autres bras.

La société se tourne alors vers l'Asie. Déjà, en 1884, elle avait employé 166 Chinois engagés pour cinq ans. Ce premier recrutement, effectué à la hâte à Singapour par un intermédiaire local, avait donné lieu à des mécomptes en raison de l'inaptitude de nombreux engagés au travail des mines. Afin d'éviter de nouveaux déboires, la société Le Nickel charge l'un de ses représentants de Nouméa, Maurice Bigillion, de se rendre en Asie pour y trouver des travailleurs. Après sept mois de démarches incessantes à Hong Kong, Shanghai, Canton, Hanoï et Haïphong, Bigillion ne parvient pas à embaucher un seul travailleur. Ce qui est possible pour une société anglaise ayant pignon sur rue dans un port chinois s'avère impossible pour un Français de passage qui veut emmener des coolies sous contrat dans un pays qu'ils ne connaissent pas. En outre, des difficultés d'ordre diplomatique,

1. NDLR - La société Le Nickel a été fondée à Paris en 1880. Elle a été contrôlée par la banque Rothschild de 1883 à 1974 . À partir de 1974, une série de restructurations a conduit à la création d'un groupe minier et métallurgique dénommé Eramet. Ce groupe est organisé en trois branches : nickel, alliages, manganèse. La société Le Nickel-sLN est donc devenue la filiale calédonienne de ce groupe dont elle est le berceau. Elle est aujourd'hui détenue à $56 \%$ par Eramet, à $34 \%$ par la STCPI (Société territoriale calédonienne de participation industrielle) et à $10 \%$ par la compagnie japonaise Nisshin Steel. Au lendemain de la Deuxième Guerre mondiale, on a pris l'habitude de la désigner sous le sigle SLN. Auparavant, cette désignation n'existait pas, on parlait de « la société Le Nickel » ou « du Nickel». 
liées au récent conflit sino-français au Tonkin (1884-1885), entravent les efforts de Bigillion. Par ailleurs, la concurrence que se livrent les colonies européennes pour obtenir des coolies vient encore compliquer sa tâche. Même à Hanoï et à Haïphong, il semble que l'administration coloniale française préfère réserver les coolies chinois disponibles aux colons d'Indochine. Et comme, de son côté, la société Le Nickel se refuse à embaucher des ouvriers sans contrat de louage pré-établi, la situation est bloquée.

En février 1891, en désespoir de cause, la société Le Nickel engage François Lütscher, un ancien agent diplomatique du consulat français de Tché Fou (Zhefu), pour prendre le relais de Bigillion. Après deux mois de tractations, Lütscher semble être sur le point d'aboutir : à l'exception de la question du salaire, il obtient l'accord du préfet du Chantoung (Shandong) pour le recrutement de 2000 à 2500 ouvriers avec un premier essai de 600 engagés. Informé que cette affaire relevait de l'État, Lütscher se rend à Pékin. Le chef du gouvernement, qui craint que les Chinois soient employés aux mêmes conditions que la main-d'œuvre pénale qu'ils doivent remplacer ou renforcer, le renvoie au préfet du Chantoung. Pris dans les arcanes de la hiérarchie administrative et politique chinoise, Lütscher doit se rendre à l'évidence : le gouvernement chinois ne repousse pas officiellement la demande du Nickel mais il augmente peu à peu ses prétentions. Après avoir tenté de recruter des travailleurs sans autorisation officielle, Lütscher jette l'éponge. Il informe la société Le Nickel qu'en " présence du mouvement dirigé contre les étrangers, il n'y avait pas de garantie pour l'exécution des contrats de main-d'œuvre, quand bien même ces contrats auraient été signés " (archives de la société Le Nickel-sLN, procès-verbal du conseil d'administration, 30 novembre 1891). Bref, ni ses efforts répétés, ni sa bonne connaissance de la Chine ne permettent à Lütscher de mieux réussir que Bigillion.

Cependant, mettant à profit ses relations au consulat japonais de Tché Fou, Lütscher a l'idée de se tourner vers le Japon. Déjà, l'année précédente, Bigillion avait tenté une démarche similaire auprès du consul du Japon en poste à Hong Kong. Des contingents de travailleurs japonais avaient effectivement déjà été recrutés quelques années auparavant en Australie et surtout à Hawaii pour la récolte de la canne à sucre. Bigillion essuie un nouveau refus : en mai 1890, la demande du Nickel est rejetée par le ministre des Affaires étrangères japonais, Aoki Shûzo. Ce dernier explique que :
« [cette] décision d'interdire l'envoi de travailleurs japonais à l'étranger est d'ordre général et ne s'applique pas à votre seul projet. » (Kobayashi Tadao, $1992: 16)$

Il ajoute que Hawaii présente un cas différent en raison d'un traité particulier. D'après Kobayashi Tadao, ce refus est dû à un faisceau de raisons : les conditions de travail et la durée de cinq ans des contrats sont jugées inacceptables car, au regard de la législation japonaise, la durée de ce type de contrat est limitée à une année ; la Nouvelle-Calédonie est mal connue des autorités japonaises qui s'inquiètent en outre de la présence du bagne.

Pourtant, l'année suivante, Lütscher est plus heureux quand il renouvelle la demande de travailleurs auprès de Nose Tatsugorô, consul par intérim du Japon à Tché Fou. Il signe un contrat portant sur un contingent de 600 engagés japonais en décembre 1891. Comment ce revirement des autorités japonaises peut-il être expliqué ? La demande de Lütscher est arrivée sur le bureau d'Aoki Shûzo le 25 novembre 1891, soit seulement quatre jours avant sa démission provoquée par un incident diplomatique survenu avec la Russie. Son successeur, Enomoto Takeaki, a des idées bien différentes sur l'intérêt que présente l'émigration japonaise.

Le parcours du nouveau ministre illustre bien l'évolution rapide du Japon vis-à-vis de l'extérieur. Occupant un rang élevé au sein de la Marine de guerre et fidèle à la famille Tokugawa qui contrôlait le shogunat depuis plusieurs siècles, il s'opposa à la restauration Meiji (1868) qui établit un régime sous l'égide de l'Empereur. Battu lors de la guerre du Boshin, il s'échappa avec une partie de la flotte et présida l'éphémère République d'Ezo établie dans l'île d'Hokkaïdo. L'année suivante, il fut emprisonné à la suite de la conquête d'Hokkaïdo par les troupes du gouvernement impérial. Gracié en 1872, il intégra la Marine impériale avec le grade de viceamiral. Deux ans plus tard, il remplit avec succès une mission diplomatique en Russie. Il occupa par la suite plusieurs postes ministériels (Marine, Communications, Agriculture et Commerce). Son parcours marque bien la réconciliation nationale qui s'opéra alors autour de l'Empereur. À travers son action ministérielle Enomoto Takeaki devint l'un des principaux promoteurs de l'émigration japonaise en direction des mers du Sud et de l'Amérique latine. En 1885, il créa une institution (Nan yô Kyôkai) destinée à collecter des informations sur le Pacifique tropical. Deux ans plus tard, étant ministre des Communications, il mit un navire du service de la 
surveillance des phares à la disposition d'une entreprise d'exploration dont l'un des principaux résultats fut la découverte de l'île d'Iwo Jima annexée par le Japon en 1889. Devenu ministre des Affaires étrangères en 1891, il imposa un département dédié à l'émigration au sein de son ministère contre l'avis du Premier ministre Matsukata Masayoshi (Peattie, 1988 : 5-8). L'action d'Enomoto Takeaki s'inscrit dans un débat qui divisa les dirigeants nippons entre une expansion dirigée vers le nord du continent asiatique, notamment Sibérie et Manchourie, et une expansion tournée vers l'océan Pacifique. Enomoto Takeaki est un marin et souhaite, comme d'autres hauts responsables japonais, voir son pays devenir une grande puissance maritime.

Parallèlement, la volonté de favoriser un large mouvement d'émigration obéit à un impératif interne au Japon. L'émigration est envisagée comme un moyen efficace de réduire la misère qui frappe de nombreuses familles paysannes. $\mathrm{La}$ teneur d'une lettre adressée à Enomoto Takeaki en juin 1892 alors que des difficultés apparaissent entre les engagés et la société Le Nickel le montre bien. Son auteur, le préfet du département dont sont originaires les engagés, écrit :

«Cette émigration était présentée comme une expérience susceptible de venir en aide aux pauvres. Mais si elle devait échouer, cela aurait des répercussions considérables sur le gouvernement. " (Kobayashi Tadao, $1992: 63$ )

Que ce soit en raison d'impératifs intérieurs ou d'ambitions extérieures, la demande de la société Le Nickel arrive donc au bon moment.

Demeure cependant la méconnaissance de la colonie française dont la mauvaise réputation liée à la présence du bagne ne manque toujours pas d'inquiéter les autorités japonaises. Le ministre Enomoto Takeaki charge Nose Tatsugorô de s'informer sur la colonie auprès de Lütscher. Ce dernier se rend à Tokyo pour poursuivre la négociation. Il y rencontre Enomoto Takeaki, auprès duquel il a obtenu une audience grâce à Joseph Sienkiewicz, le ministre plénipotentiaire de France au Japon. Lütscher décrit l'archipel calédonien en insistant sur la salubrité de son climat. Il prend quelques libertés avec la réalité quand il affirme que le travail des mines n'est ni pénible ni dangereux en raison de l'exploitation à ciel ouvert. De même quand il assure que les ouvriers japonais bénéficieront d'un statut similaire à celui des colons libres. Il appuie sa démonstration sur la protection établie par un arrêté qui règle la condition des engagés immigrés dans la colonie. En réalité, cet arrêté daté du 26 mars
1874 place les engagés dans une condition inférieure à celle des colons libres. Quoi qu'il en soit, le ministre donne son accord de principe le 10 novembre 1891. La correspondance confidentielle produite par Kobayashi Tadao (1992) montre bien que le consul et le ministre étaient favorables à cet accord dès le départ des négociations.

C'est donc à l'initiative d'une société privée, la société Le Nickel, qu'est organisée la première immigration de travailleursjaponais en NouvelleCalédonie. Comme nous venons de le voir, ce recrutement a nécessité deux années de recherches, de démarches et de négociations pour aboutir. Il est alors prévu que ce contingent de 600 hommes soit le premier d'une série de convois réguliers.

\section{Le premier contingent d'engagés japonais (1892- 1897)}

Le 6 janvier 1892, 599 hommes débarquent sur la plage de Thio, le principal centre minier de la société Le Nickel. Ils ont embarqué à Nagasaki dix-huit jours auparavant. L'un des engagés est mort au cours de la traversée. Le contingent est accompagné d'un médecin, d'un interprète, d'un surveillant général, Ono Yaichi, et de ses adjoints. Leur recrutement a été mené par une compagnie japonaise formée ad hoc: la Nihon Yoshisa Imin Kaisha. La compagnie est chargée d'assurer la liaison entre l'employeur et les engagés afin de protéger les droits de ces derniers. Pour sa part, la société Le Nickel confie sa représentation au Japon à un négociant anglais de Tokyo nommé James. Comme pour les autres catégories d'engagés immigrés, ce recrutement entre dans le cadre juridique de l'arrêté du 26 mars 1874. Les contrats sont de cinq ans.

Qui sont ces premiers engagés? À la suite de l'accord intervenu en 1891, les préfets de Nagasaki et de Kumamoto (île de Kyushu) sont invités par le gouvernement à encourager le recrutement en assurant aux populations concernées que les conditions de cette émigration seraient meilleures que celles de l'émigration dirigée vers Hawaii depuis quelques années. Effectivement, après un premier essai sans lendemain en 1868, l'émigration japonaise vers Hawaii est devenue massive à partir de 1885. En 1890, déjà plus de 12000 Japonais, soit $14 \%$ de la population hawaïenne, y sont recensés contre seulement cent seize en 1884. Ce fort mouvement s'est accompagné de difficultés dues aux antagonismes ethniques qui en ont découlé, antagonismes attisés par les planteurs afin de mieux contrôler la main- 
d'œuvre à leur disposition (De Deckker, 1994 : 184). Ces difficultés connues au Japon inquiétaient les candidats au départ, d'où l'insistance du gouvernement pour les rassurer.

Par ailleurs, pour de nombreux paysans pauvres, obtenir un contrat en Nouvelle-Calédonie pouvait leur permettre d'amasser le pécule indispensable pour acquérir une parcelle une fois de retour au pays. En effet, la population japonaise, après avoir longtemps stagné, a connu un fort accroissement, passant de 35 millions au milieu du XIX ${ }^{\mathrm{e}}$ siècle à 51 millions en 1912. Or, cet essor démographique s'est produit dans un pays où le fractionnement de la surface agricole utile était déjà très poussé, aggravant un processus général de paupérisation largement amorcé. C'était notamment le cas pour de nombreux paysans de l'île de Kyushu qui, en outre, avait été touchée par des conditions climatiques désastreuses pour l'agriculture en 1891. Le recrutement de paysans habitués au dur travail des champs intéressait la société Le Nickel. La condition requise pour être embauché était d'être un agriculteur âgé de 25 à 30 ans. Cependant, Le Nickel qui attendait impatiemment cette nouvelle source de bras, a poussé à ce que le recrutement soit accéléré. Faute d'un nombre suffisant d'agriculteurs parmi les candidats, environ cinquante hommes sans aucune aptitude pour le travail des mines, des petits commerçants ou des étudiants déclassés, sont embauchés à la hâte pour compléter le convoi. La plupart des recrutés sont originaires de la commune d'Amakusa, une île située à l'ouest de Kyushu.

Des problèmes surviennent rapidement. Après quelques jours, de nombreux engagés refusent de travailler et demandent à retourner dans leur pays. Le blocage est total. Les grévistes se plaignent de la dureté des conditions de travail. La société Le Nickel réplique en appliquant à la lettre les peines prévues par la réglementation. En cas d'insubordination et de refus de travail, le commissaire de l'immigration saisi par l'engagiste peut prononcer une peine de huit à soixante jours d'atelier disciplinaire à l'encontre des engagés. Ces derniers sont alors employés sans solde et sous surveillance à des travaux d'utilité publique. Ils peuvent être punis en cas d'indiscipline. Les sanctions s'échelonnent de la manière suivante : un fer à un pied, un fer aux deux pieds, isolement dans une salle sans lumière, arrêt de la fourniture de viande prévue dans la ration. En outre, la société Le Nickel applique une autre disposition de la réglementation locale en infligeant aux grévistes une retenue de deux jours de salaire par jour non travaillé (arrêté du 26 mars 1874, articles 39, 51 à 55).
Apparemment, une quarantaine d'engagés a été ainsi envoyée dans les pénitenciers. Les plus lourdes peines touchent six engagés considérés comme les meneurs de la grève : ils passent un mois en atelier disciplinaire (Kobayashi Tadao, $1992: 62,71)$.

De nombreux engagés écrivent à leurs proches pour décrire les conditions de travail qu'ils endurent et les punitions qu'ils encourent. Ils leur demandent d'agir afin d'être rapatriés au plus vite. La teneur de leurs lettres soulève une grande inquiétude à Amakusa. L'affaire remonte à la diète impériale. En juin 1892, le député Kodama Nakaji s'adresse au gouvernement pour savoir si les rumeurs ayant trait aux mauvais traitements que recevraient les émigrés en NouvelleCalédonie sont fondées. Si oui, il lui demande ce qu'il compte faire.

En fait, la veille de cette demande, la société d'émigration Yoshisa a déjà reçu l'ordre de faire un rapport sur la situation. Pour ce faire, elle s'appuie elle-même sur un rapport que lui a adressé le surveillant général Ono Yaichi :

« Le 8 février dernier, tous les émigrés ont demandé de suspendre leur travail et de rentrer dans leur pays pour les deux raisons suivantes : la difficulté du travail et l'état des chemins trop raides menant à leurs lieux de travail. À cette occasion [...] onze émigrés ont été envoyés en prison à Nouméa [...]. [Les émigrés] se sont maintenant bien habitués au climat et connaissent de mieux en mieux les Français, ce qui leur permet de travailler sans problème. »" (Kobayashi Tadao, $1992: 62$ )

Les versions des événements présentées par la Yoshisa et les familles des engagés diffèrent très sensiblement. Devant les problèmes que pourrait engendrer cette situation pour la pérennité du mouvement d'émigration que souhaite développer le gouvernement, le préfet de Kumamoto prévient le ministre Enomoto Takeaki qu'il a chargé un agent de son administration, Senda Ichijuro, d'aller enquêter sur place.

Dans son rapport de mission, l'inspecteur Senda Ichijuro présente une situation nuancée. Il est choqué par le caractère de certaines mesures répressives (la détention en atelier disciplinaire en particulier) et par les carences du service de santé du Nickel. Mais il souligne aussi les torts de certains des engagés vis-à-vis de l'employeur. Aux yeux de Senda, le principal problème est la direction du personnel et les pressions exercées sur les engagés.

Le gouvernement japonais ne peut intervenir en ce qui concerne les mesures répressives prévues par la loi française. Il s'en remet à la Yoshisa pour négocier avec Le Nickel afin d'améliorer la 
situation. Mais le mal est fait, les relations entre l'employeur et la plupart des engagés sont irrémédiablement mauvaises. De son côté, la société Le Nickel a intérêt à voir partir cette main-d'œuvre en raison du retournement du marché qui entre en récession l'année même de l'arrivée des engagés japonais à Thio.

L'effectif en état de travailler s'amenuise rapidement. Cinquante huit engagés, dont l'incapacité au travail des mines est admise par la société d'émigration, sont renvoyés au Japon dès le mois d'août 1892. De nombreux autres sont atteints par le béribéri. En mai 1894, il ne reste plus qu'une centaine de Japonais sur les chantiers de la société Le Nickel. Les derniers rentrent dans leur pays en février 1898.

L'expérience n'est pas renouvelée immédiatement, non tant à cause des difficultés qu'a occasionnées le recrutement de 1892, mais simplement parce que la société dispose alors d'un nombre suffisant de travailleurs. Pour répondre à la reprise du marché qui s'esquisse en 1898, elle compte, dans un premier temps, utiliser à plein les condamnés dont elle dispose encore et dont elle parvient à tirer un travail plus productif.

Ce premier recrutement de Japonais en Nouvelle-Calédonie a donc soulevé des difficultés pour les deux parties concernées. D'un côté, les engagés n'acceptent pas les conditions de travail qui leur sont imposées et leur gouvernement s'inquiète de voir ainsi sa volonté de favoriser l'émigration de ses ressortissants être entravée. De l'autre, la société Le Nickel, qui comptait avoir enfin trouvé les bras qui lui manquaient, se trouve confrontée à une maind'œuvre pugnace. Cependant, le départ prématuré de la plupart des engagés est plutôt heureux pour la société en raison du retournement de la conjoncture à partir de 1892 . Elle attend que la reprise du marché du nickel soit bien établie pour de nouveau s'intéresser à la main-d'œuvre japonaise.

\section{Une deuxième vague massive à partir de 1900}

En juin 1899, le directeur parisien du Nickel André Marchand annonce au conseil d'administration qu'il a repris contact avec James qui réside désormais en Angleterre. Il lui a demandé de se mettre en rapport avec les compagnies japonaises d'émigration et les compagnies maritimes afin de leur demander les conditions les plus favorables pour un nouveau recrutement de Japonais.

Malgré les démarches les plus actives, les accords des autorités japonaises et françaises tardent à arriver. Sur le plan local, le gouverneur Paul Feillet fait rapidement savoir à la société qu'il ne fait aucune opposition à l'entrée d'ouvriers japonais dans la colonie. Face aux résultats décevants de la colonisation libre qu'il appelle de ses vœux et qu'il fonde sur la culture du café, Feillet voit dans l'exploitation minière un moyen de relancer ce mouvement. Pour cela, il a besoin de l'appui de la société Le Nickel avec laquelle il a élaboré un projet d'établissement de colons mineurs à Kouaoua (Bencivengo, 2010 : 865-870). Paradoxalement, il autorise donc la société à recruter des Japonais afin de mener à bien son projet de colonisation libre. En revanche, il faut attendre le mois de janvier 1900 pour que le gouvernement japonais autorise le recrutement. Ce dernier exige et obtient de Paris que ses ressortissants soient considérés comme des travailleurs libres. C'est là le point capital de la négociation. Le ministre des Colonies Albert Decrais s'en explique ainsi :

« C'est grâce à notre adhésion à la demande formée par le gouvernement japonais en vue de l'application du droit commun à ses nationaux qu'ont pu être aplanies les difficultés soulevées tout d'abord au sujet du recrutement d'une main-d'œuvre que la NouvelleCalédonie considérait comme indispensable au développement de ses exploitations. " (Bulletin officiel de la Nouvelle-Calédonie, 21 janvier 1901)

Le contrat avec la société d'émigration japonaise, la Tôyô Imin Gôshi Kaisha, est signé le 28 février 1900. Toutefois, des complications surgissent, retardant l'arrivée des travailleurs. Par exemple, le gouvernement japonais exige que les hommes soient payés 38 francs au lieu de 32 francs par mois. En outre, la compagnie de navigation réclame $9 £$ au lieu de $7 £$ pour le transport de chaque homme. Le directeur du Nickel expose les raisons pour lesquelles la société accepte ces nouvelles exigences :

« Bien que ces conditions dépassent de beaucoup celles portées au contrat, le conseil est d'avis qu'il faut les accepter, car il importe avant tout de fournir aux mines en Nouvelle-Calédonie une main-d'œuvre qui permette d'assurer la production à la consommation des usines, de constituer un stock et de donner à la société une avance sur les sociétés qui pourraient se constituer pour l'exploitation des mines de nickel. » (Archives de la société Le Nickel-SLN, procès-verbal du conseil d'administration, 11 juin 1900)

Les propos du ministre et ceux du directeur du Nickel montrent bien que l'intérêt économique a pesé lourd dans leurs décisions. L'exploitation des mines exige une main-d'œuvre nombreuse et 
efficace. La société Le Nickel doit en passer par là pour maintenir sa suprématie locale.

De juin 1900 à mars 1901, un millier de travailleurs sous contrat arrivent à Thio. Ce recrutement inaugure une série de convois se succédant en 1905, 1911, 1914 puis 1919 à la date de renouvellement des contrats. Au total, un peu plus de trois mille travailleurs sont ainsi introduits en Nouvelle-Calédonie par la société Le Nickel. S'y ajoutent par ailleurs 1500 hommes engagés par la maison Ballande qui contrôle la société des Hauts Fourneaux de Nouméa, principale concurrente locale du Nickel. Leur origine géographique s'élargit : la majorité des engagés recrutés à partir de 1900 viennent de l'île de Honshu. Ce sont des hommes jeunes: les deux tiers d'entre eux sont âgés de 21 à 30 ans à leur arrivée (Palombo, 2002: 174). D'après Kobayashi Tadao la plupart sont des paysans. Seul le convoi de 1905 comprend une soixantaine de femmes.

Cependant, la situation se dégrade rapidement entre la société et les engagés. Dès octobre 1900, un conflit éclate à propos de l'insuffisance des soins médicaux. Quelques mois plus tard, en avril 1901, une grève se déclare à Thio. Les trois quarts des Japonais cessent le travail. Apparemment, comme leurs prédécesseurs de 1892, ils n'ont pas été suffisamment informés sur le travail qui les attendait et pour lequel ils n'étaient pas préparés. Leur principale revendication est la réduction de la durée de leur contrat. Le mouvement est durement réprimé par les forces de l'ordre envoyées par le gouverneur qui craint un exode massif des engagés vers Nouméa. Les grévistes, dont la subsistance dépend directement de la société Le Nickel qui pourvoit à leur nourriture dans le cadre des contrats, sont quasiment à l'état de famine et n'ont d'autre choix que de reprendre leur travail au Nickel ou de trouver rapidement un autre employeur. Le travail reprend le 7 mai 1901. Les meneurs sont arrêtés, puis relâchés le 18 mai suivant. Cent dix-huit Japonais quittent alors les chantiers de Thio. Après la reprise du travail, de nombreux grévistes - ceux qui n'acceptent pas les conditions qui leur sont imposées - ne reviennent pas et le mouvement des évasions se poursuit lentement. Face à cette véritable hémorragie, la société Le Nickel semble être assez démunie. Les engagés japonais ne sont plus soumis à l'arrêté de 1874 . Le seul recours du Nickel est de menacer ceux qui seraient tentés d'employer les évadés. Par exemple, en mai 1901, la société fait paraître l'avis suivant dans la presse locale :

«La société Le Nickel prévient le public que les Japonais à son service sont engagés par des contrats individuels régulièrement passés pour une durée de cinq années, qui a commencé en juin, août, novembre, décembre 1900 et mars 1901. Par conséquent, elle est propriétaire pour toute cette période de temps du travail des Japonais et les personnes qui lui déroberaient cette propriété en employant ceux-ci seront poursuivies par toutes les voies légales. » (La France australe, 8 août 1901)

En outre, une partie significative des effectifs japonais est tenue écartée des chantiers par les maladies. Certains engagés sont déjà malades ou affaiblis à leur arrivée et nombre d'entre eux supportent mal les dures conditions du travail des mines. En février 1901, deux cents Japonais sont touchés par une épidémie d'influenza. S'ajoute la peste qui s'est déclarée dans la colonie en 1900, et qui réapparaît en août 1901 à Thio où elle provoque la mort de deux Kanak et d'un Japonais. Mais la principale cause des décès est le béribéri. À la suite de mesures sanitaires concernant l'alimentation, la situation se stabilise à partir de 1903.

Une nouvelle vague d'évasions se produit après l'arrivée du convoi de 1911. Pour Miho Gorô, l'attaché du consulat du Japon de Sydney envoyé en Nouvelle-Calédonie pour enquêter sur place, la faiblesse des salaires des engagés du Nickel en regard de ce qui leur est proposé par d'autres exploitants miniers est la principale raison des évasions :

«Le salaire de la société Le Nickel est de 40 francs par mois plus la nourriture. Par contre, les autres entreprises recrutent à plus de 3 francs par jour et la main-d'œuvre du chemin de fer est à 3,50 francs par jour au même tarif que les Européens, c'est-à-dire près de 100 francs par mois ; même si la nourriture n'est pas offerte, la différence est énorme. Dans ces conditions, il semble très difficile d'empêcher les Japonais de déserter et de refuser l'offre des autres entreprises. 》 (Kobayashi Tadao, 1992 : 99)

La principale mesure prise par la société d'émigration pour tenter d'endiguer les évasions est de mettre les frais de voyage aller-retour à la charge de l'engagé et non plus de l'employeur. En contrepartie, les salaires doivent être augmentés (mais ce n'est guère le cas pour Le Nickel) et la durée des contrats est ramenée à quatre ans.

Le recrutement de travailleurs japonais cesse après l'arrivée du convoi de 1919 qui n'amène qu'une centaine d'hommes. La société Le Nickel se tourne dès lors vers la main-d'œuvre tonkinoise jugée plus docile. Il semble bien que cet arrêt soit aussi la conséquence d'un changement de la politique du Japon en matière d'émigration. Philippe Palombo remarque que les engagés qui rentrent alors définitivement au Japon 
appartiennent globalement à la classe des mobilisables. Il cite les propos d'un journaliste rapportés par le gouverneur de NouvelleCalédonie dans sa correspondance avec le ministre des Colonies :

« Il semble qu'en rentrant au Japon, ils obéissent à un mot d'ordre. Le gouvernement [japonais] aurait-il besoin de ses ressortissants pour satisfaire sans délai ses vues sur la Mandchourie, la Sibérie et la Chine orientale ? (Palombo, 2002 : 162)

\section{Les représentants d'une grande puissance en devenir}

Les Japonais présentent donc un cas à part dans les mouvements d'immigration de travail qui ont été organisés en Nouvelle-Calédonie jusqu'à la Deuxième Guerre mondiale. Leur statut et les négociations auxquelles il a donné lieu en sont la marque principale. En 1892, ils sont soumis à l'arrêté du 26 mars 1874. Cet arrêté, qui a servi de référence pour les textes ultérieurs réglementant les différents courants d'immigration de travail qui se sont succédé, mais aussi pour le statut des Kanak engagés, a été notamment pris dans le but de mettre fin aux abus auxquels a donné lieu le recrutement des Néo-Hébridais. Il prévoit le contrôle des conditions de recrutement, établit une visite médicale, fixe la durée des contrats, réglemente les conditions de vie (rations alimentaires, logement, habillement), les horaires de travail et les modalités du rapatriement. Les engagés n'ont cependant pas le statut de travailleurs libres. Ils dépendent de leur engagiste qui détient leur livret. Leur circulation dans la colonie est strictement limitée. Enfin, cet arrêté autorise l'établissement de taxes destinées à couvrir les dépenses du service de l'immigration (arrêté du 26 mars 1874, article 80).

Lors des négociations ouvertes en 1899 dans le but de reprendre le recrutement, c'est sur cette question que les autorités japonaises refusent de céder. C'est qu'entre-temps le Japon a durci les mesures de contrôle et de protection concernant ses émigrants. Une loi japonaise sur l'émigration du 7 avril 1896 prévoit que « Nul ne peut émigrer sans l'autorisation du gouvernement » (article 1) et réglemente sévèrement l'activité des agents d'émigration (Bulletin officiel de la NouvelleCalédonie, dépêche ministérielle du $1^{\text {er }}$ août
1896). Cette évolution est peut-être entre autres à mettre en relation avec les difficultés rencontrées par les émigrants japonais à Hawaii et avec l'agitation qui se manifeste alors contre eux en Californie où leur nombre s'accroît dans les années $1890^{2}$. En Nouvelle-Calédonie, les élus locaux s'opposent à ce que les engagés japonais soient considérés comme des travailleurs libres. La crainte que cette situation d'égalité suscite au sein de la population blanche n'est pas la seule raison de ce refus. Cette disposition prive le budget local des taxes prévues par l'arrêté de 1874 au moment où l'État réduit sa participation dans les dépenses locales (Buttet, 1996 : 369-376). Mais rien n'y fait. L'affaire est directement négociée de gouvernement à gouvernement sans tenir compte des protestations qui s'élèvent dans la colonie. C'est à Paris qu'est prise la décision que les nouveaux engagés devront « être placés, à la Nouvelle-Calédonie, non sous le régime spécial institué par l'arrêté du 26 mars 1874, mais sous le régime du droit commun et qu'ils seront traités sur le même pied que les travailleurs européens "». Hormis l'intérêt économique déjà évoqué plus haut, le ministre des Colonies Decrais présente un argument d'ordre diplomatique :

« Je ne saurais donc aujourd'hui revenir sur cette question sans parler des embarras certains que nous créerait un changement d'attitude à cet égard : nous devons être affermis dans la discussion prise vis-à-vis des Japonais par les événements qui se sont déroulés récemment en Extrême Orient. » (Bulletin officiel de la Nouvelle-Calédonie, 21 janvier 1901)

Déjà, le ton de la remarque de Decrais dénote probablement les difficultés qu'éprouvent les diplomates français dans leurs discussions avec le gouvernement japonais. D'autres témoignages confirment l'attitude souvent pointilleuse, voire hautaine, des responsables japonais vis-à-vis de leurs interlocuteurs occidentaux. Ainsi Maurice Pelletier qui négocie en 1910 pour le compte d'une autre société implantée en NouvelleCalédonie (la société des Hauts-Fourneaux de Nouméa), s'agace-t-il des retards incessants et de la multiplication des démarches qu'il doit effectuer (Palombo, 2002: 81). On peut penser que cette attitude exprime notamment la volonté affichée du Japon depuis le début de l'ère Meiji d'être considéré comme une puissance à part entière traitant d'égal à égal avec les puissances occidentales.

2. La situation des immigrés japonais en Californie s'aggrave après la victoire du Japon sur la Russie en 1905. Une association, la Japanese and Korean Exclusion League, est alors constituée dans le but d'obtenir des mesures de ségrégation à leur encontre. Ils sont l'objet de mesures vexatoires de la part de l'état de Californie au lendemain du grand tremblement de terre de 1906. Il faut attendre 1907 et la conclusion du Gentleman's Agreement entre l'État fédéral et le gouvernement japonais pour que cette crise soit en partie résolue. 
Par ailleurs, l'exigence formulée par le Japon reflète bien la nouvelle place qu'il occupe dans le concert des nations, tout au moins dans l'aire Asie-Pacifique. L'événement auquel le ministre Decrais fait allusion est la révolte des Boxers ${ }^{3}$ (1900) au cours de laquelle le Japon a pris part à la répression aux côtés des puissances occidentales. C'est à partir de sa victoire sur la Chine en 1895 que la position du Japon s'est affirmée dans la région. Devenu partenaire des puissances occidentales dans les affaires chinoises, il parvient à faire réviser des traités inégaux qui le plaçaient dans une position inférieure. De même, il entend bien protéger ses ressortissants et leur assurer un statut qui les place en position d'égalité avec les Européens. Il est donc inacceptable pour lui qu'ils soient soumis à une réglementation visant des travailleurs venus d'autres colonies (Java et Indochine notamment), et donc assimilés à des indigènes aux droits inférieurs à ceux des colons. C'est aussi l'exigence de voir les émigrants être bien traités qui explique le souci constant avec lequel les autorités japonaises contrôlent les conditions de leur transport maritime, de leurs logements et de leur nourriture. Les navires doivent présenter des caractéristiques précises telles que le nombre et la taille des hublots afin d'assurer un confort minimum aux émigrants. La société Le Nickel fait aménager des salles de bains spéciales dans les logements qu'elle attribue aux Japonais. La nourriture est directement importée du Japon pour satisfaire aux habitudes alimentaires des engagés. Ce dernier point soulève deux types de difficultés. Il apparaît que la part de la viande prévue dans les rations est trop faible et la carence en protéines qui en résulte donne lieu à une épidémie de béribéri. Quand le médecin de la société Le Nickel obtient que la ration soit enrichie en protéines, il se heurte à l'opposition de certains engagés qui refusent de changer leurs habitudes alimentaires. Par ailleurs, l'importation des denrées japonaises est très mal perçue dans la colonie car elle constitue un manque à gagner pour l'agriculture locale. Elle renforce le sentiment négatif à l'égard de la société qui est accusée d'exploiter les richesses du pays sans que ce dernier n'en retire de bénéfices.

D'autres dispositions des contrats confirment la qualité de travailleurs libres des engagés japonais. Leur salaire est bien plus élevé que celui des autres catégories d'immigrés. Ils sont accompagnés et représentés par un agent de la compagnie d'émigration. Mais si le gouvernement japonais veut protéger ses ressortissants, il veut aussi les surveiller et s'assurer qu'ils ne ternissent pas l'image que le pays veut présenter au monde. C'est aussi là le rôle du surveillant général qui exerce une véritable autorité sur les engagés. De même, les inspecteurs envoyés sur place s'attachent à décrire le comportement de leurs compatriotes. Les engagés japonais doivent donc manifester leur patriotisme. Leur attitude est particulièrement surveillée lors du premier conflit mondial au cours duquel le Japon s'engage aux côtés de l'Entente dès août 1914, juste avant de commencer sa campagne de prise de possession de certaines îles de la Micronésie.

Cette image de ressortissants d'une puissance qui pourrait souhaiter s'imposer dans la région s'inscrit en creux dans les attaques dont ils sont victimes. L'arrivée des travailleurs japonais inquiète les travailleurs libres européens dont la présence sur les chantiers du Nickel se réduit à l'encadrement. Cela au moment même où la colonie connaît un long marasme économique en cette première décennie $\mathrm{du} \mathrm{xx}^{\mathrm{e}}$ siècle. Les attaques contre la présence des Japonais se multiplient au fur et à mesure de l'accroissement du nombre d'engagés. Les invectives n'ont rien que de très classique. La presse locale, souvent directement inspirée par les journaux australiens, publie des articles sur «La conspiration du silence », "La protection de la main-d'œuvre blanche », « Le péril japonais », « L’invasion des Jaunes », « La Nouvelle-Calédonie livrée aux Jaunes » (La France australe, 29 mars 1909, 9 et 13 janvier 1911, Le Bulletin du Commerce, 14 février et 13 juin 1914). Si ces articles relèvent d'un racisme ordinaire que l'on retrouve vis-àvis des autres ethnies, ils sont aussi le signe d'une véritable inquiétude face à la montée de la puissance japonaise, notamment après sa victoire sur la Russie en 1905, abondamment commentée dans la presse locale. Les écrits ayant trait aux engagés japonais ont une tonalité différente de ceux consacrés aux Tonkinois et aux Javanais qui, venant de colonies, sont assimilés aux indigènes. Pour les Japonais, il est question d'une « invasion », d'un " péril ». La présence des engagés japonais réputés généralement efficaces et relevant d'un statut équivalent à celui des Blancs, est considérée comme une véritable menace. Nul doute que le comportement des travailleurs japonais vienne alimenter cette psychose.

C'est que ce comportement relève d'une véritable modernité dans un pays où les employeurs, ceux des mines particulièrement, ont pris

3. Cette révolte a été initiée par une société secrète chinoise dont le symbole était un poing fermé, d'où le nom de "Boxeurs" donné par les Occidentaux. Les Boxeurs furent à l'origine d'un mouvement xénophobe et nationaliste qui éclata en 1898, à la suite de la défaite infligée à la Chine par le Japon en 1895 et des exigences des puissances européennes. 


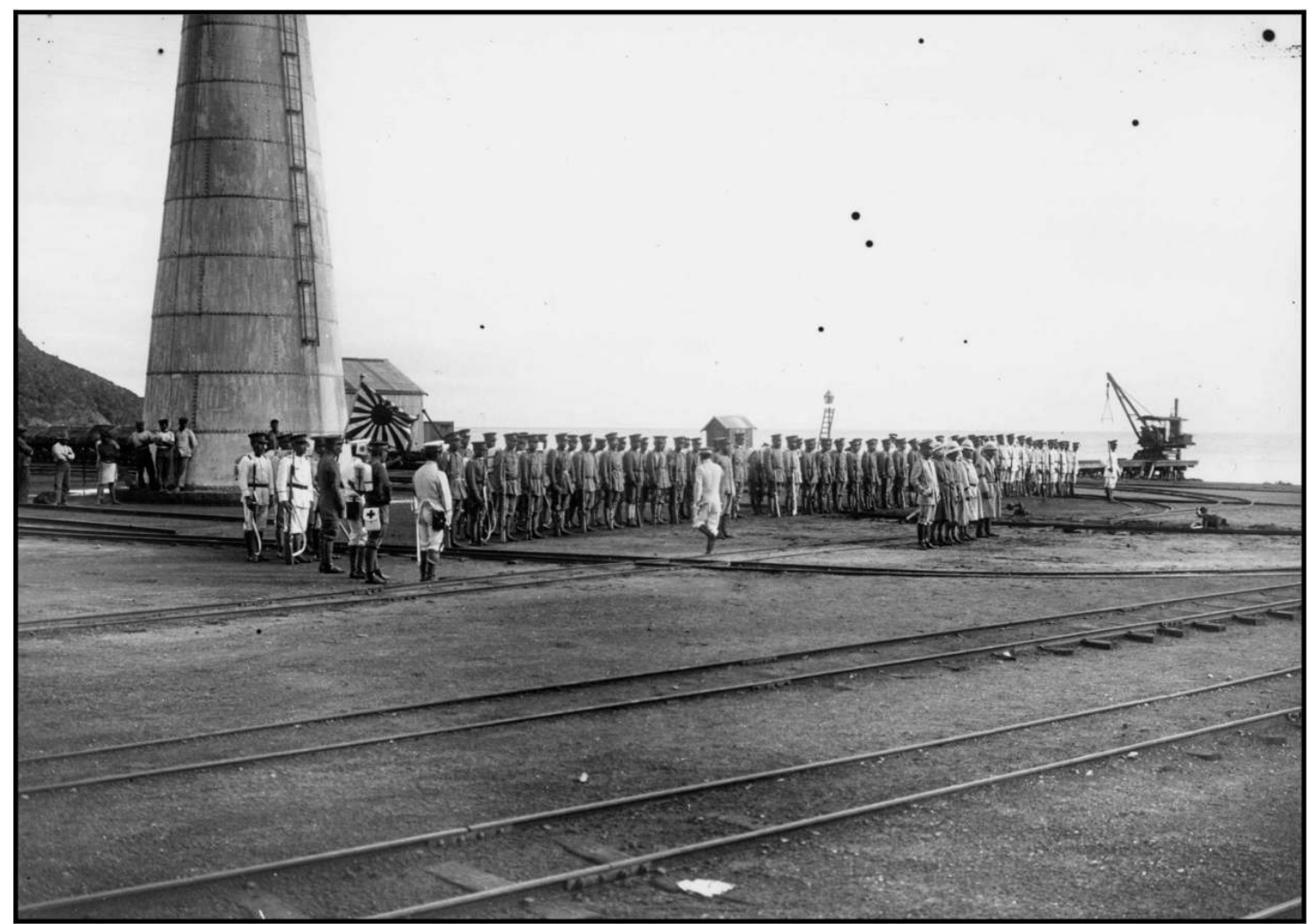

Рното 1. - « Fête japonaise » à Thio, le 31 octobre 1916 (C archives de la Nouvelle-Calédonie, fonds Maxime Meyer, 2Ph7, 607). Cette photographie a été prise au milieu des installations de transport, de stockage et d'embarquement du minerai qui se trouvaient dans la plaine de Thio-Mission. À gauche, on aperçoit le pied de l'un des pylônes du système qui permettait la mise en tas du minerai, à droite on peut distinguer la grue du grand wharf

l'habitude de disposer d'une main-d'œuvre docile. Premier acte: les grèves; majoritaires parmi les engagés en 1901, elles constituent un fait sans précédent dans la colonie. Elles montrent aussi la cohésion des ouvriers japonais dans leurs revendications. Deuxième acte : les évasions ; s'estimant libérés de leurs obligations par des conditions de travail jugées inacceptables, de nombreux engagés japonais brisent unilatéralement leur contrat pour trouver un emploi mieux rémunéré ou s'établir à leur compte. S'ajoutent les engagés qui au terme de leur contrat préfèrent rester en Nouvelle-Calédonie. À partir de 1913, date de la promulgation dans la colonie d'une nouvelle convention de commerce passée en 1911 entre la France et le Japon, les Japonais ont désormais le droit de demeurer dans la colonie (Angleviel, 2000 : 73). Certains restent au Nickel comme ouvriers vraiment libres, dégagés de tout contrat du type de celui avec lequel ils sont venus travailler. Nombre d'entre eux parviennent à des postes de maîtrise et font une longue carrière au sein de l'entreprise. D'autres se reconvertissent et utilisent leur pécule pour s'installer, notamment comme com- merçants. Après la fin des convois en 1919, une communauté japonaise demeure ainsi dans la colonie. À la suite du déclenchement de la Guerre du Pacifique, les Japonais sont arrêtés et déportés en Australie, d'où ils seront reconduits vers le Japon après 1945 (Tsuda, 2012). En 1941, selon un recensement effectué en vue de leur expulsion, ils sont 1104 à vivre dans la colonie, dont 57 femmes. Extrêmement rares sont ceux qui ont pu alors rester en Nouvelle-Calédonie (Palombo, 2002 : annexe XVII).

Enfin, le recrutement des Japonais présente une nouveauté de taille. Pour la première fois, des non Européens, venant d'un pays dégagé de toute dépendance à l'égard des puissances occidentales, viennent travailler en NouvelleCalédonie. Cette immigration marque le premier pas de l'intégration de l'économie calédonienne dans l'espace Pacifique. Jusque-là, tous les travailleurs immigrés étaient des habitants d'un pays dépendant plus ou moins directement d'une puissance européenne et dont la population était soumise à un statut d'indigène. Â partir de 1900, les Japonais sont des travailleurs qui exercent leurs droits et parviennent à résister aux 
employeurs qui n'ont finalement qu'une emprise relative sur eux. Les difficultés rencontrées par la société Le Nickel pour enrayer les évasions le montrent bien. Par ailleurs, jusque-là, l'économie calédonienne n'était, pour l'essentiel, en connexion qu'avec l'économie française et européenne. Durant l'immigration japonaise, des liens économiques se tissent avec la puissance régionale montante. Avec la Première Guerre mondiale et la coupure quasi totale des relations maritimes entre la colonie et la métropole, les sociétés minières locales cherchent de nouveaux débouchés. C'est pendant la Grande Guerre que les premières expéditions de minerais de nickel vers le Japon sont effectuées (trois convois en 1916). Cette diversification des exportations perdure après la fin des hostilités alors que la sidérurgie japonaise connaît un développement rapide. Pourvoyeur de main-d'œuvre jusqu'à la guerre, le Japon devient un client de la principale activité économique de l'archipel.

Il devient aussi un investisseur. Dans les années 1920, plusieurs sociétés minières à capitaux japonais sont constituées dans la colonie. Les deux plus importantes sont la société Le Fer et la Société minière de l'Océanie. Avec la montée des tensions internationales, ces sociétés sont parfois considérées comme des « paravents » des ambitions japonaises en Nouvelle-Calédonie (Palombo, 2002 : 325). De nouvelles dispositions $\mathrm{du}$ droit minier local (les autorisations personnelles de miner) ont pour but de limiter le développement du domaine minier de ces sociétés. Une fois la guerre déclarée, leurs biens sont confisqués. À partir de 1951, le Japon redevient un client important. Cette évolution aboutit par exemple à des participations croisées quiexpliquent qu'aujourd'huila sociétéLe Nickel soit détenue à hauteur de $10 \%$ par une société métallurgique japonaise, la Nisshin Steel.

\section{Conclusion}

À l'échelle calédonienne, l'immigration japonaise présente donc des caractères tout à fait originaux par rapport aux autres mouvements migratoires qu'a connus la colonie jusqu'à la Deuxième Guerre mondiale. Contrairement aux Européens venus librement tenter leur chance ou à ceux amenés contre leur gré comme forçats il n'est pas question de participer au peuplement pénal ou libre de la colonie. Il est prévu qu'à l'issue de leur contrat, les engagés japonais retournent dans leur pays. Contrairement aux Néo-Hébridais, aux Javanais et aux Tonkinois qui sont assimilés à des indigènes, les Japonais sont des travailleurs libres dont les droits sont défendus par des agents qui les accompagnent. Les négociations ardues avec les autorités nipponnes pour l'obtention d'engagés constituent un signe tangible de la volonté du Japon de traiter d'égal à égal avec les puissances coloniales occidentales.

À l'inverse, la nécessité pour le Japon de trouver des espaces où il peut envoyer sa population surnuméraire souligne l'une de ses faiblesses. Le décollage industriel n'en est qu'à ses débuts et l'économie nipponne n'est pas encore en mesure d'absorber le choc démographique des campagnes. Quelles que soient les précautions dont il entoure les recrutements, il doit se résoudre à placer une partie de ses émigrants sous l'autorité de compagnies étrangères.

Une fois arrivés dans la colonie, les engagés font montre d'une grande modernité face au travail. Ils n'hésitent pas à s'opposer à des conditions qu'ils jugent inacceptables par des grèves dures. Ils osent rompre unilatéralement leur contrat afin de faire valoir leurs compétences auprès d'autres employeurs, créant de fait un véritable marché du travail dans un pays où la masse des travailleurs étaient jusqu'alors étroitement soumise à des contrats qui les plaçaient dans une position juridique inférieure.

À l'échelle de l'Asie-Pacifique, cette situation est moins originale. Elle est à mettre en relation avec les mouvements de colonisation que lance le Japon vers la Chine et la Micronésie. Elle est aussi à comparer avec l'immigration japonaise à Hawaii, où l'on distingue l'immigration de « qualité » des Japonais de celle des Chinois qui les ont précédés. La politique menée par le Japon en Nouvelle-Calédonie concernant ses ressortissants est la même que celle appliquée pour les migrations vers Hawaii.

Par ailleurs, l'établissement du mouvement migratoire entre le Japon et la NouvelleCalédonie marque l'entrée active de la colonie dans un espace auquel elle appartient sur le plan géographique mais dans lequel elle demeurait isolée économiquement et politiquement. Bien sûr, elle entretient des relations commerciales avec l'Australie et la Nouvelle-Zélande voisines, notamment pour les denrées périssables. Mais l'essentiel de ses relations sont strictement dirigées vers la métropole et l'Europe. C'est notamment le cas des compagnies minières, qui non seulement envoient tous leurs minerais et leurs produits métallurgiques vers l'Europe, mais s'y approvisionnent aussi largement en machines, matériaux pondéreux, matériaux de construction et équipements divers. L'immigration japonaise est donc le premier contact direct 
de la colonie avec la puissance montante régionale. Ces liens se renforcent quand, à la faveur de la Première Guerre mondiale, les compagnies minières calédoniennes, coupées de leurs bases européennes, trouvent un Japon en plein essor industriel capable d'absorber une partie de leur production restée sur le carreau des mines. Ce lien va perdurer jusqu'à nos jours. Bref, l'immigration japonaise en Nouvelle-Calédonie constitue la première étape vers la connexion de l'économie calédonienne avec l'espace économique Asie-Pacifique.

$\mathrm{Au}$ terme de cette courte étude principalement fondée sur les résultats d'une thèse consacrée à l'histoire d'une société minière et métallurgique, il subsiste de nombreuses questions.

Il faudrait connaître de façon beaucoup plus fine la situation des engagés avant leur départ. Certains ont-ils déjà connu une expérience similaire? Quelles sont les raisons précises de leur engagement? Quelles sont les relations qu'ils entretiennent avec leurs proches pendant la durée de leur contrat ? Quelle influence peuventils avoir dans les recrutements suivants? L'importance des évasions doit aussi être éclairée. Leur caractère massif et précoce peut laisser penser que nombre des engagés ont déjà décidé de rompre rapidement leur contrat avant même d'avoir débarqué en Nouvelle-Calédonie. Quelles sont les relations qu'ils entretiennent avec ceux qui les ont précédés? Quel est leur devenir après l'évasion? Pour ceux qui ont honoré leur contrat, il serait tout aussi intéressant de connaître les motivations de ceux qui font le choix de continuer dans l'entreprise comme travailleurs complètement libres ou de s'installer à leur compte. Choisir de quitter son pays pour s'installer définitivement dans un pays lointain, à la culture très différente, où une partie de la population est notoirement hostile à la présence des Japonais, est une démarche lourde de sens qui mérite d'être analysée. Et qu'en est-il de ceux qui font au contraire le choix de rentrer au Japon ? Quelles sont les conséquences de leur séjour en Nouvelle-Calédonie sur leur parcours ultérieur? La fin des convois après 1919 est-elle seulement due aux employeurs calédoniens qui désirent trouver une main-d'œuvre plus docile? N'est-elle pas aussi l'expression de la volonté $\mathrm{du}$ Japon de mettre fin à cette émigration de travailleurs dont il peut avoir besoin sur son territoire pour son propre développement industriel?

Enfin, en Nouvelle-Calédonie comme ailleurs dans le Pacifique, les Japonais ont souvent été accusés d'espionnage. Dans quelle mesure le Japon a-t-il envisagé l'intérêt stratégique que pourrait revêtir la présence de milliers de ressortissants dans des contrées qui pourraient bien devenir le lieu de luttes ultérieures dans la domination de l'espace Pacifique?

\section{BIBLIOGRAPHIE}

ANGLEVIEL Frédéric, 2000. De l'engagement comme « esclavage volontaire ». Le cas des Océaniens, Kanak et Asiatiques en Nouvelle-Calédonie (18531963), Journal de la Société des Océanistes 110, pp. $65-81$.

Archives DE LA Nouvelle-Calédonie, 1915 (31 octobre). Fonds Maxime Meyer, 2Ph7, 602.

Archives De L'OUtre-Mer, 1883. Rapport Courmeaux, série géographique, carton 180 .

Archives DE LA SOCIÉTÉ LE NICKEL-SLN, 1891 (30 novembre). Procès-verbal du conseil d'administration.

—, 1900 (11 juin). Procès-verbal du conseil d'administration.

Bencivengo Yann, 2010. La société Le Nickel. Une entreprise au cœur de la naissance de l'industrie du nickel (1880-1914), thèse de doctorat d'histoire, Université de Paris 1 Sorbonne-Panthéon.

Boubin-Boyer Sylvette, 2001. De la Première Guerre mondiale en Océanie. Les guerres de tous les Calédoniens (1914-1919), thèse de doctorat en anthropologie historique, Université de NouvelleCalédonie.

Le Bulletin du Commerce, 1914 (14 février). L'invasion des Jaunes, p. 1.

_ 1914 (13 juin). La Nouvelle-Calédonie livrée aux Jaunes, p. 1.

BulLETIN OfFICIEL DE LA NOUVELLE-CALÉDonie, 1896 ( $1^{\mathrm{er}}$ août). Dépêche ministérielle.

—, 1901 (21 janvier).

Buttet Catherine, 1996. Histoire d'un échec ? Mise en valeur et pouvoirs publics en NouvelleCalédonie (1870-1914), thèse de doctorat en histoire, Université d'Aix-en-Provence.

CORre Bruno, 1989 (octobre). Les migrations en Nouvelle-Calédonie à travers les Archives du service de l'Immigration, Publications de l'Université du Pacifique, vol. 1, fasc. 3 et 4, Nouméa, actes du colloque CORAIL Migration et Identité, pp. 11-16.

De Deckker Paul (éd.), 1994. Le peuplement du Pacifique et de la Nouvelle-Calédonie au $X I X^{\mathrm{e}}$ siècle (1788-1914). Condamnés, Colons, Convicts, Coolies, Chân Dang, Paris, L'Harmattan, actes du colloque universitaire international.

DelAthiÈRE Jerry, 2012. L'arrestation des Japonais de La Foa, Farino et Sarraméa (déc. 1941- fév. 1942), Bulletin de la Société d'études historiques de la Nouvelle-Calédonie 171, pp. 22-53. 
Duus Peter, Ramon H. Myers and Mark R. Peattie (eds), 1989. The Japanese Informal Empire in China, 1895-1937, Princeton, Princeton University Press.

IDoux Joseph, 1967. Sociétés s'étant occupées dans le territoire de la Nouvelle-Calédonie de métallurgie du nickel, Nouméa, société Le Nickel.

Kobayashi Tadao, 1992. Les Japonais en NouvelleCalédonie, histoire des émigrés sous contrat, Nouméa, Publications de la Société d'études historiques de la Nouvelle-Calédonie.

LA France Australe, 1901 (8 août).

_, 1909 (29 mars). La conspiration du silence, p. 1.

—, 1911 (9 janvier). La protection de la main-d'œuvre blanche, p. 1.

—, 1911 (13 janvier). Le péril japonais, p. 1.

PAlombo Philippe, 2002. La présence japonaise en Nouvelle-Calédonie (1890-1960). Les relations économiques entre le Japon et la NouvelleCalédonie à travers l'immigration et l'industrie minière, thèse de doctorat en histoire, Université de la Nouvelle-Calédonie.

PeAtTIE Mark, 1988. Nan'yō. The Rise and Fall of the Japanese in Micronesia, 1885-1945, Honolulu, University of Hawaii Press.

Raulet-AKaza Keiko, 20 I 2. Yaichi Ono (1847-1893) et la première émigration japonaise en NouvelleCalédonie, Bulletin de la Société d'études historiques de la Nouvelle-Calédonie 171, pp. 2-21.

SHINEBERG Dorothy, 1991. Nouméa no good, Nouméa no pay, Journal of Pacific History 26, 2, pp. 187-205.

Totman Conrad, 2005. A History of Japan, Oxford, Blackwell Publishing.

TsudA Mutsumi, 2006. Feu nos pères. Les émigrants japonais en Nouvelle-Calédonie, Nouméa, Comité du projet.

_, 2012. Âmes errantes. Le destin brisé des émigrants d'Okinawa en Nouvelle-Calédonie, Nouméa, Madrépores. 